\title{
Optical immersion of mid-infrared LEDs and photodiodes for gas sensing applications
}

\author{
H.R.Hardaway*a , C.T.Elliott ${ }^{\mathrm{a}}$, N.T.Gordon ${ }^{\mathrm{a}}$, J.G.Crowder ${ }^{\mathrm{b}}$ \\ a'QinetiQ, St Andrew's Road, Malvern, Worcestershire, WR14 3PS, UK; ${ }^{b}$ Physics \\ Department, Heriot-Watt University, Edinburgh, EHI4 4AS, UK
}

Keywords: Mid-infrared, gas sensing, optical immersion, LEDs, photodiodes.

\section{ABSTRACT}

The high gains in performance predicted for optical immersion are difficult to achieve in practice due to total internal reflection at the lens/detector interface. By reducing the air gap at this interface optical tunnelling becomes possible and the predicted gains can be realised in practical devices. Using this technique we have demonstrated large performance gains by optically immersing mid-infrared heterostructure InAlSb LEDs and photodiodes using hyperspherical germanium lenses. The development of an effective method of optical immersion that gives excellent optical coupling has produced a photodiode with a peak room temperature detectivity (D*) of $5.3 \times 10^{9} \mathrm{cmHz}^{1 / 2} \mathrm{~W}^{-1}$ at $\lambda_{\text {peak }}=5.4 \mu \mathrm{m}$ and a $40^{0}$ field of view. A hyperspherically immersed LED showed a 5-fold improvement in the external efficiency, and a 3fold improvement in the directionality compared with a conventional planar LED for $f / 2$ optical systems. The incorporation of these uncooled devices into a White cell produced a $\mathrm{NO}_{2}$ gas sensing system with 2 part-per-million sensitivity, with an LED drive current of $<5 \mathrm{~mA}$. These results represent a significant advance in the use of solid state devices for portable gas sensing systems.

\section{INTRODUCTION}

High detectivity, room temperature photodiodes operating in the 3-8 $\mu \mathrm{m}$ region, and complementing LEDs, are in great demand for mid-infrared (MIR) gas sensing systems ${ }^{1}$. Gases with fundamental absorption features in the MIR include $\mathrm{CH}_{4}(3.3 \mu \mathrm{m}), \mathrm{CO}_{2}(4.2 \mu \mathrm{m}), \mathrm{CO}(4.7 \mu \mathrm{m}), \mathrm{N}_{2} \mathrm{O}(4.8 \mu \mathrm{m}), \mathrm{NO}_{2}$ $(6.3 \mu \mathrm{m}), \mathrm{SO}_{2}(7.3 \mu \mathrm{m})$ and $\mathrm{H}_{2} \mathrm{~S}(7.6 \mu \mathrm{m})$. Existing MIR gas sensing systems use relatively low sensitivity thermal detectors such as thermopiles or pyroelectrics $\left(\mathrm{D}^{*} \approx 1 \times 10^{8} \mathrm{cmHz}^{1 / 2} \mathrm{~W}^{-1}\right)$, with incandescent sources. The higher sensitivity and intrinsic safety (particularly in explosive environments) of the solid state components, together with better stability and longevity, favour their use in such applications.

The sensitivity of large area (typically $1 \mathrm{~mm}^{2}$ ) photodiodes is still limited by the Johnson noise generated by the relatively large series resistance despite the progress made towards improving the $\mathrm{R}_{0} \mathrm{~A}$ of MIR photodiodes. In addition, the poor impedance matching to external amplifiers further reduces the sensitivity of a working detector. Optically immersing the detectors using a lens of the required shape and refractive index offers a solution to both these problems. The area magnification of the lens allows the actual area of the detector to be reduced which gives an inherent sensitivity gain whilst improving the impedance matching.

Optically immersion also improves the performance of the LEDs. The external efficiency of planar LEDs is limited by the effects of total internal reflection at the semiconductor-air interface: this is particularly severe in InSb where $\mathrm{n}=4$. By optically coupling the light from the planar LED into a suitably shaped lens light which would be totally internal reflected exists the curved lens surface at near normal incidence.

The use of immersion lens in this manner is well established, however, the development of suitable immersion wavelength for the mid-infrared has yet not been clearly demonstrated. 


\subsection{The benefits of immersed detectors}

\section{THEORY}

The $\mathrm{D}^{*}$ of a Johnson noise limited unbiased photodiode with amplifier is described by:

$$
D_{p e a k, \lambda}^{*}=\frac{\eta_{\mathrm{det}} \cdot \lambda}{1.24} \cdot \frac{R_{d}}{\sqrt{4 k_{B} T R_{d}+4 k_{B} T R_{s}+i_{a}^{2}\left(R_{d}+R_{s}\right)^{2}+e_{a}^{2}}} \cdot A_{a}^{1 / 2}
$$

where $\eta_{\text {det }}$ is the quantum efficiency, $\lambda$ is the wavelength, $A_{a}$ is the apparent area, $R_{d}$ is the dynamic resistance, $R_{s}$ is the series resistance, and $i_{a}$ and $e_{a}$ are the amplifier current and voltage noise, respectively. The $\mathrm{R}_{\mathrm{d}} \mathrm{A}$ of the unbiased heterostructure photodiodes described here is approximately $2 \times 10^{-3} \Omega \mathrm{cm}^{2}$ at $300 \mathrm{~K}$, which for a $1 \mathrm{~mm}^{2}$ photodiode with an internal efficiency of $\eta_{\text {det }} \approx 40 \%$, corresponds to a Johnson limited $\mathrm{D}^{*} \approx 6 \times 10^{8} \mathrm{cmHz}^{1 / 2} / \mathrm{W}$ at $\lambda=5 \mu \mathrm{m}$ when connected to a noiseless amplifier. When the large area, low impedance, photodiodes required for gas sensing are connected to the input of a low noise amplifier $\left(\mathrm{e}_{\mathrm{a}}=4 \times 10^{-10} \mathrm{VHz}^{-1 / 2}, \mathrm{i}_{\mathrm{a}}=1.2 \times 10^{-11} \mathrm{AHz}^{-1 / 2}\right)$, the combined effects of amplifier noise and series resistance $\left(\mathrm{R}_{\mathrm{s}}=2 \Omega\right)$ reduce the $\mathrm{D}^{*}$ significantly to $3 \times 10^{7} \mathrm{cmHz}^{1 / 2} \mathrm{~W}^{-1}$.

By optically immersing the detectors using a suitable lens, the $\mathrm{D}^{*}$ is improved ${ }^{2}$ by reducing the actual area of the device whilst maintaining the same apparent area due to the lens magnification. The reduction in the actual area of the devices also greatly improves impedance matching to the external electronics. In the ideal case (with no low index intermediate layer) optical immersion using a hemispherical lens of refractive index $n_{\text {lens }} \approx n_{\text {detector }}$ magnifies the effective area by $n_{\text {lens }}^{2}$. This area gain increases the $D^{*}$ by a factor $n_{\text {lens }}$ and $R_{d}$ by a factor $n_{\text {lens }}{ }^{2}$ compared with an unimmersed detector with the same apparent area.

Further improvements in $\mathrm{D}^{*}$ can be gained by immersing the detector using an aplanatic hyperspherical lens (or Weierstrass sphere): a spherical lens which has been truncated a distance $t=r / n_{\text {lens }}$ from the centre. This lens has an $\mathrm{n}_{\text {lens }}{ }^{4}$ area gain and thus $\mathrm{n}_{\text {lens }}{ }^{2}$ improvement in the $\mathrm{D}^{*}$ compared with an unimmersed device, however, this improvement in the $\mathrm{D}^{*}$ is accompanied by a reduction in the half angle field of view (FOV) which is reduced to $\sin ^{-1}\left(1 / \mathrm{n}_{\text {lens }}\right)$. The maximum theoretical $\mathrm{D}^{*}$ for our InAlSb device, optically immersed using a germanium $\left(n_{\text {lens }}=4.0\right)$ hyperspherical lens, is $9.0 \times 10^{9} \mathrm{cmHz}^{1 / 2} \mathrm{~W}^{-1}$, which is reduced to $\mathrm{D}^{*}=6.9 \times 10^{9} \mathrm{cmHz}^{1 / 2} \mathrm{~W}^{-1}$ when the noise components from $\mathrm{R}_{\mathrm{s}}, \mathrm{i}_{\mathrm{a}}$ and $\mathrm{e}_{\mathrm{a}}$ are included. This significant improvement is due to the inherent $\mathrm{n}^{2}$ gain in the $\mathrm{D}^{*}$, the increase in $\mathrm{R}_{\mathrm{d}} / \mathrm{R}_{\mathrm{s}}$, and the improved matching to the amplifier

\subsection{The benefits of immersed LEDs}

Optical immersion can also improve the performance of planar LEDs ${ }^{3}$. Progress towards reducing non-radiative recombination in narrow gap LEDs has increased the internal efficiency ${ }^{4}$, however, the external efficiency of planar LEDs is still limited by the total internal reflection (TIR) at the semiconductor-air interface: this is particularly severe in MIR devices where the refractive index is high, e.g. $\mathrm{n}_{\mathrm{InSb}}=4.0$. Surface texturing ${ }^{5}$ and resonant cavity structures ${ }^{6}$ can improve the external efficiency of near transparent LEDs by increasing the fraction of light that exits at near normal incidence. However, these methods are unsuitable for bulk emitting devices described here due to the high absorption coefficient of the emitting region.

If effective optical coupling between the LED and the immersion lens is achieved, radiation which would otherwise be total internally reflected is transmitted from the planar LED into the lens, and subsequently exits the spherical lens-air interface at near normal incidence. Optical immersion gives an $\mathrm{n}_{\text {lens }}{ }^{2}$ improvement in the radiant power $(\phi)$, if the refractive indices of the LED and lens are well matched, and the lens radius is large enough to ensure that all radiation exits the lens at near normal incidence (typically $\left.\mathbf{r}_{\text {lens }}>\mathbf{r}_{\text {det }} \cdot n_{\text {lens }}\right)$. Although the radiant power is increased, the LED's radiance $(L=d \phi / d A d \Omega)$ remains constant because the increase in the radiant power and reduction in the emission angle $(\Omega)$ is negated by the increase in the effective emitting area (A). A significant advantage from hyperspherical optical immersion is that the radiation is emitted with improved directionality, i.e. larger $\mathrm{f} / \mathrm{no}$. to match the following optics. The current needed for a given power is also reduced. 


\subsection{The importance of optical coupling}

The potential benefits of immersing detectors and LEDs are strongly dependent on efficient optical coupling of radiation across the lens/device interface for incident angles $0>\theta_{\mathrm{i}}>\pi / 2$. In the absence of a high refractive index cement layer, transmission across the interface at $\theta_{\mathrm{i}}>\theta_{\mathrm{TR}}$ is only possible if the separation between the lens and device is small enough to allow the propagation of the evanescent wave mode. Using the matrix methods similar to those described by Heavens ${ }^{7}$, the calculated transmission coefficient between the lens and the detector separated by an air gap of distance, $\mathrm{d}$, as a function of incident angle, $\theta_{\mathrm{i}}$, is shown in figure 1 for $\lambda=6 \mu \mathrm{m}$. From these calculations we can see that a lens/device separation of $d<\lambda /\left(4 . n_{\text {lens }}\right) \approx 0.2 \mu \mathrm{m}$ is required for high (greater than $90 \%$ ) normal incidence responsivity, large detector FOV and efficient radiation extraction from the LED's. Achieving this small separation using a suitable immersion lens is technically challenging.

\section{EXPERIMENTAL DETAILS}

We have fabricated InAsSb heterostrucuture diodes from MBE grown layers, which have been described elsewhere ${ }^{8,9}$ with the layer structure: $\mathrm{n}^{+} \mathrm{InSb}$ substrate; $\mathrm{n}^{+}\left(\mathrm{Si}: 3 \times 10^{18} \mathrm{~cm}^{-3}\right) \mathrm{InSb} ; \mathrm{p}^{+}\left(\mathrm{Be}: 2 \times 10^{18} \mathrm{~cm}^{-}\right.$ $\left.{ }^{3}\right) \mathrm{InSb} ; \mathrm{p}^{+}\left(\mathrm{Be}: 2 \times 10^{18} \mathrm{~cm}^{-3}\right) \mathrm{In}_{0.85} \mathrm{Al}_{0.15} \mathrm{Sb}$; near-intrinsic $\mathrm{InSb}$ active region $(3 \mu \mathrm{m}) ; \mathbf{n}^{+}\left(\mathrm{Si}: 3 \times 10^{18} \mathrm{~cm}^{-3}\right)$. This structure is advantageous as it prevents carriers thermally generated outside the active region from contributing to the noise current. The drift of thermally generated electrons from the p-region is prevented by the wider-band gap, strained, $\mathrm{In}_{0.85} \mathrm{Al}_{0.15} \mathrm{Sb}$ barrier, whilst the thermal generation of holes in the $\mathbf{n}^{+}$ region is suppressed by degenerate doping. The inclusion of the barrier and the degenerate doping also buffer the intrinsic region from carriers thermally generated at the contacts. The $\mathbf{n}^{+}-\mathrm{p}^{+}$interface provides a low-resistance, tunnel contact to the p-type region of the diode. The LED structure is similar to the detector structure with the exception of a shorter near-intrinsic active region of $1.5 \mu \mathrm{m}$. In forward bias the shorter active region combined with the containing properties of the barriers increases the carrier concentration in the active region. This is thought to saturate the SRH non-radiative channels.

From these epilayers, $82 \mu \mathrm{m}$ diameter mesas were wet etched and $\mathrm{Cr} / \mathrm{Au}$ contacts were deposited using a method that allows good optical contact between the top $\mathrm{n}^{+}$epilayer and the lens.

Undoped, monocrystalline germanium was the preferred lens material because of its high refractive index, ease of machining, and transparenc $y$ in the MIR range.

Both the detector and LED were immersed using hyperspherical lenses with a $2.5 \mathrm{~mm} \pm 0.1 \mathrm{~mm}$ radius of curvature, $r$, and a $0.56 \mathrm{~mm} \pm 0.05 \mathrm{~mm}$ cylindrical extension, $t$. This value of $t$ was specified to avoid the severe aberrations introduced when $t$ exceeds $r / n_{\text {lens }}=0.625 \mathrm{~mm}$, whilst still retaining a high lens magnification.

To achieve good optical contact between the lens and the diodes, the two were aligned and held together in a specially designed jig, which applied sufficient force to achieve the minimal separation required without damaging the diode. The lens was permanently fixed to the top surface of the mesa diode by introducing (by capillary action) cyanoacrylate adhesive between the etched region surrounding the mesa and the lens flat. The device current-voltage (IV) characteristics were measured before and after immersion to confirm that the lens fixing process did not degrade the electrical characteristics. 

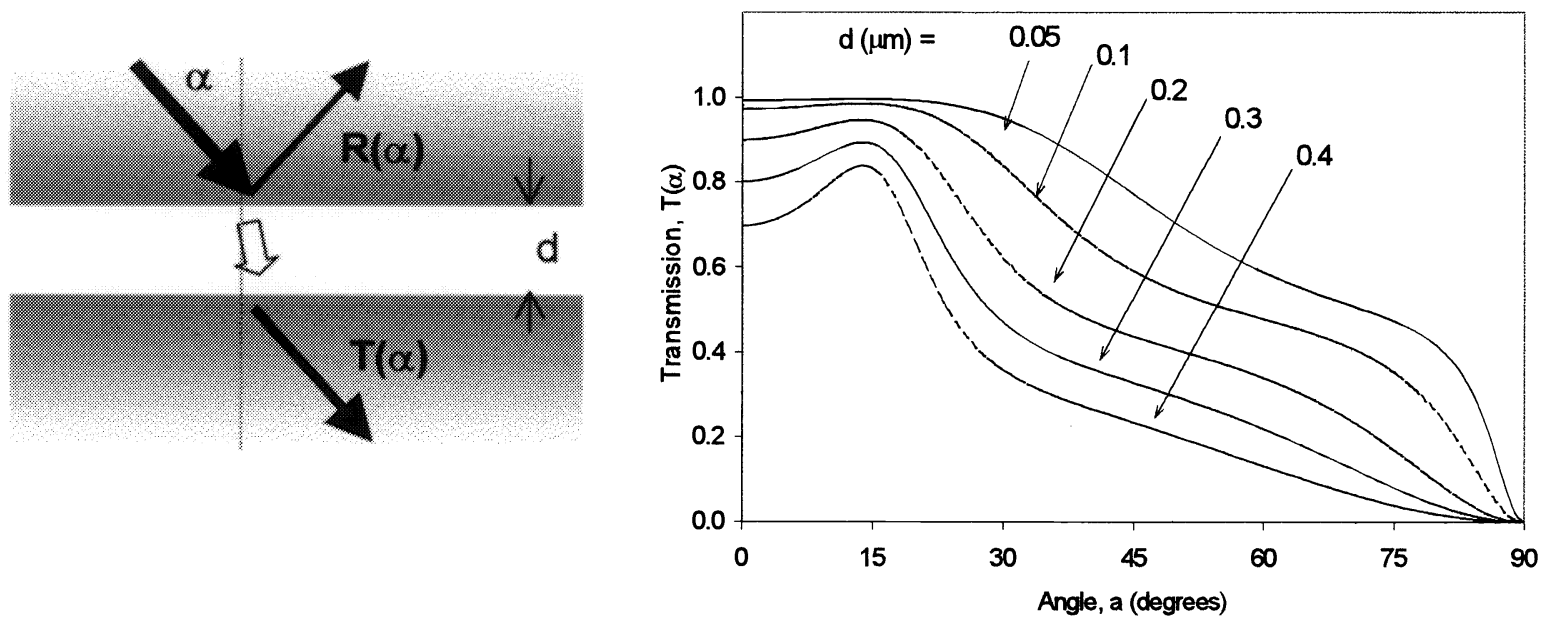

Figure 1: The calculated transmission coefficient from the Ge lens to the InSb device separated by an air gap of thickness, $d$, as a function of angle of incidence at the Ge-Air interface, $\alpha$.

\section{RESULTS AND DISCUSSION}

\subsection{Detectors}

The photodiode $R_{0}$ was $36 \Omega$ at $300 K,\left(R_{d}=32 \Omega\right.$ and $\left.R_{s}=4 \Omega\right)$ and a corresponding $R_{0} A=1.7 \times 10^{-3}$ $\Omega \mathrm{cm}^{2}$. The magnified detector image was clearly visible using a thermal imaging camera, and the 12.1-fold linear magnification of the lens gave an effective detecting area of $\mathrm{A}_{\mathrm{a}}=0.8 \mathrm{~mm}^{2}$. Figure 2 shows the detector's spectral response measured using a transmission-corrected FTIR spectrometer. The peak responsivity of $1.36 \mathrm{AW}^{-1}$ at $\lambda=5.4 \mu \mathrm{m}$ corresponds to a quantum efficiency of $\eta_{\operatorname{det}}=32 \%$. When compared with an unimmersed device, $\eta_{\text {det }}$ falls more rapidly with decreasing wavelength due to the decreasing transmission across the lens/air/detector interface for shorter wavelengths. The small absorption feature at $\lambda=5.6 \mu \mathrm{m}$ is due to the presence of adhesive in the optical path at the edge of the detector.

Figure 3 shows the variation in the detector response as it was rotated in front of a near parallel radiation from a chopped blackbody source a half cone angle of $2^{\circ}$. The high relative responsivity at normal incidence and the $20^{\circ}$ half angle FOV indicate good optical coupling between the lens and the detector. Figure 3 also shows the results for a similar device with poor optical contact at the lens-air interface which reduces the FOV and responsivity. Noise measurements obtained using a spectral analyser confirmed the noise above $60 \mathrm{~Hz}$ was equivalent to the Johnson noise, $\mathrm{n}_{\text {Johnson }}=\left[4 \mathrm{R}_{\mathrm{d}} \mathrm{kT}\right]^{1 / 2}=7.7 \times 10^{-10}$ V.Hz ${ }^{-1 / 2}$ for the unbiased detector.

From the peak responsivity, noise and area measurements we obtain a $\mathrm{D}^{*}{ }_{\text {peak } 300 \mathrm{~K}}$ of $5.3 \times 10^{9}$ $\mathrm{cm} . \mathrm{Hz}^{1 / 2} / \mathrm{W}$ at $\lambda=5.4 \mu \mathrm{m}$, which reduces slightly to $4.0 \times 10^{9} \mathrm{~cm} \cdot \mathrm{Hz}^{1 / 2} / \mathrm{W}$ due to series resistance and amplifier noise. This 2 -fold improvement in the $\mathrm{D}^{*}$ compared with previously immersed devices ${ }^{10}$ is due to: improved optical coupling between the lens and the detector, accurate alignment of the detector and lens, and improved understanding of the required lens dimensions. 


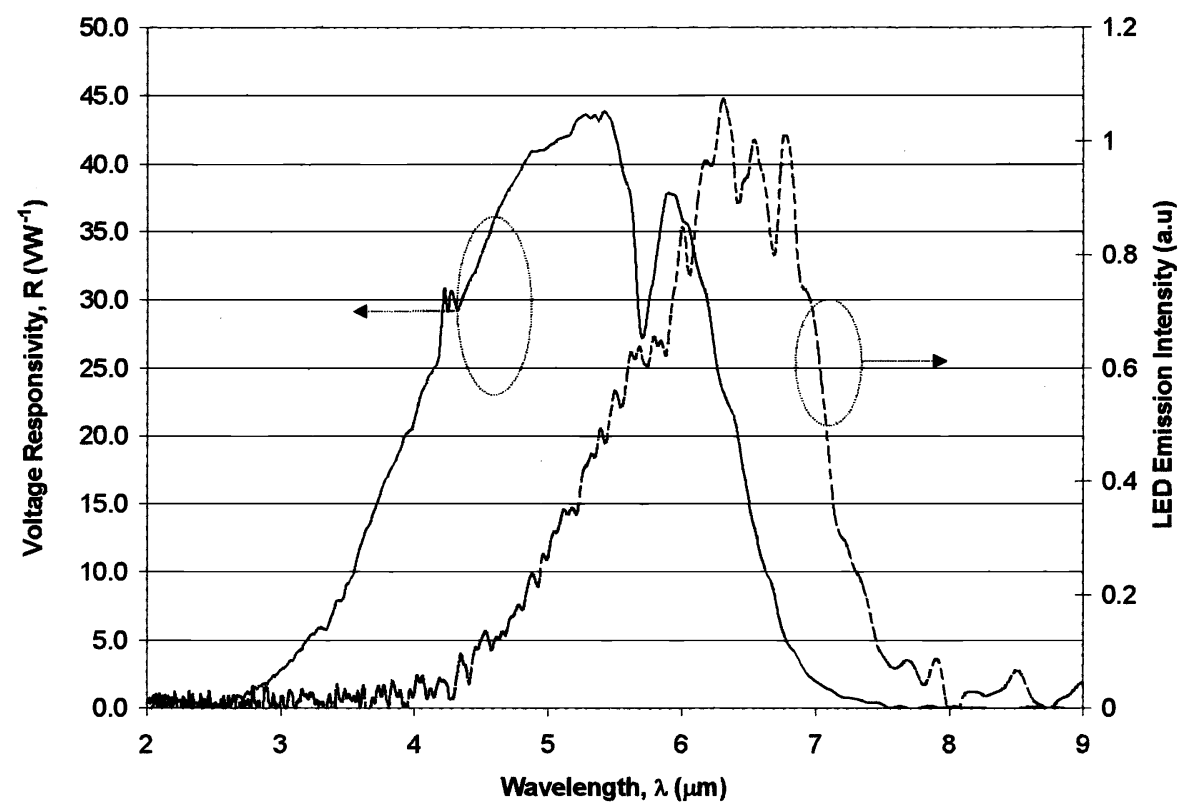

Figure 2: The immersed photodiode responsivity (solid) and immersed LED emission (dashed) as a function of wavelength, $\lambda$, at $T=300 \mathrm{~K}$.

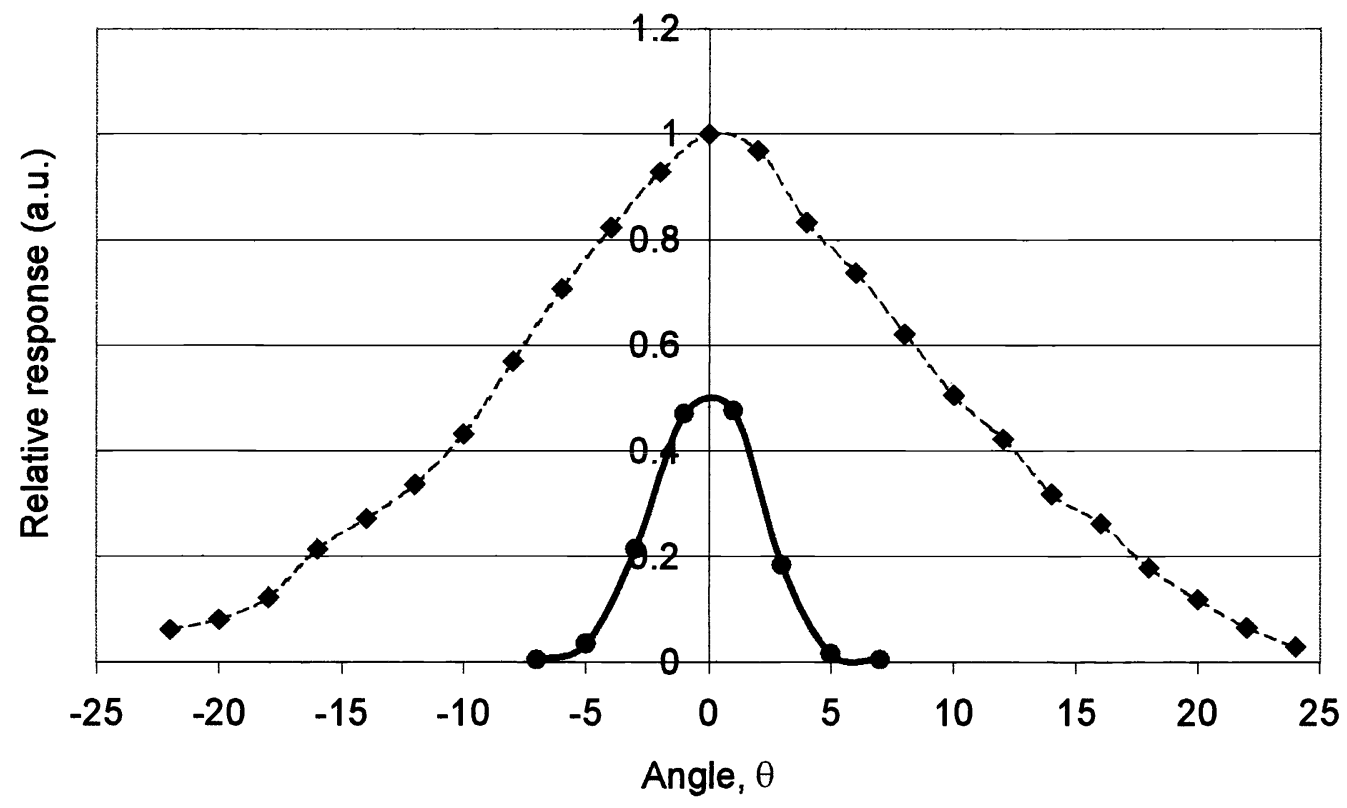

Figure 3: The variation in detector's relative response when rotated by an angle, $\alpha$, for a well immersed (diamonds) and poorly immersed (circles) photodiode. 


\subsection{LEDs}

The LED's electroluminescence spectrum (see figure 2) was measured by focusing its emission into the FTIR spectrometer using an anti-reflection coated $\mathrm{CaF}_{2}$ lens, and employing a cooled $(77 \mathrm{~K})$ cadmium mercury telluride (CMT) photoconductive detector. The peak emission at $300 \mathrm{~K}$ was at $\lambda_{\text {peak }}=6.6 \mu \mathrm{m}$ as expected from the near-intrinsic $\mathrm{InSb}\left(\mathrm{E}_{\mathrm{g}, 300 \mathrm{~K}}=180 \mathrm{meV}\right)$ emitting region. The radiant power and spatial distribution of the LED's emission was measured using a calibrated CMT photoconductor mounted on an

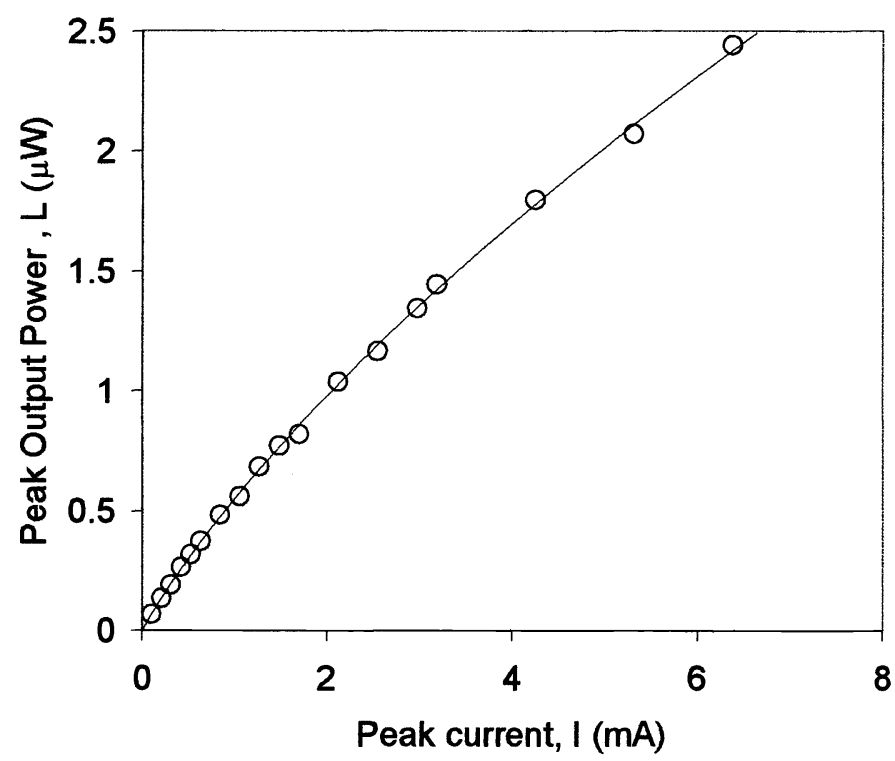

Figure 4: LI curve for an optical immersed LED. The sub-linear dependence is due to increasing Auger recombination at high current densities.

$\mathrm{x}$-y stage. The total power from the LED at $\mathrm{I}=4.5 \mathrm{~mA}(50 \%$ duty cycle) was $1.8 \mu \mathrm{W}$, which corresponds to an external efficiency of $\eta_{\text {ext }}=4.0 \times 10^{-4}$ W/A at a current density of $85 \mathrm{Acm}^{-2}$. Compared with a $1 \mathrm{~mm}^{2}$ unimmersed LED this represents a 5-fold improvement in $\eta_{\text {ext }}$, and is further evidence of good optical coupling between the lens and the diode. The luminescence-current (LI) curve for these LEDs up to $I_{\text {peak }}=7 \mathrm{~mA}$ is shown in figure 4 . The sub-linear trend in the LI curve indicates a fall in the device efficiency with increasing current. This reduction in efficiency is thought to be due to increasing Auger recombination due to increasing carrier density $\left(5 \mathrm{~mA} \sim 100 \mathrm{~A} / \mathrm{cm}^{2}\right)$ in these small area devices.

In addition to the improved external efficiency, the improved directionality of the hyperspherically immersed LED gives an additional 3-fold improvement in the power emitted into $\mathrm{f} / 2$ compared with an unimmersed Lambertian device. The combination of the higher power and improved directionality of these immersed LEDs gives a 15-fold improvement in the LED performance in optical system with $\mathrm{f} / 2$ optics.

\subsection{Gas Sensing Capability}

The immersed photodiodes and LEDs were incorporated into a gas sensing system based on a White cell $(\sim \mathrm{f} / 4)$ with a $4 \mathrm{~m}$ path length. With both devices operating at room temperature, and an LED drive current $\mathrm{I}_{\mathrm{LED}}=4 \mathrm{~mA}$ ( $50 \%$ duty cycle), the system demonstrated $2 \mathrm{ppm} \mathrm{NO}$ concentration sensitivity $^{11}$ with a 10 second integration period. Previous measurements on unimmersed LEDs in this system required $\mathrm{I}_{\mathrm{LED}}>1$ $\mathrm{A}^{12}$, and a InSb detector cooled to $-20^{\circ} \mathrm{C}$. Achieving this sensitivity with low drive currents and without any detector cooling is a clear demonstration of the benefits of immersing heterostructure LEDs and detectors. 


\section{CONCLUDING REMARKS}

The development of a practical method of optically immersing InSb photodiodes and LEDs has produced a significant improvement in the performance of both. The $\mathrm{D}^{*}$ of the photodiodes was increased by a factor of $\sim 100$ with a sufficiently large field of view for most applications. The improvement in the LEDs external efficiency and directionality show clearly the benefit of immersing the sources using this method. The combined improvements of these devices to detect $\mathrm{NO}_{2}$ with minimal power requirements and no cooling clear demonstration of the benefits of optical immersion.

\section{References}

${ }^{1}$ S.D.Smith, A.Vass. P.Bramley, J.G.Crowder, and C.H.Wang, IEE Proc. Optoelectron., 144266 (1997)

${ }^{2}$ R.Clark Jones, Applied Optics, 1607 (1962)

${ }^{3}$ C.H.Gooch, Injection Electroluminescent Devices, p.58 (John Wiley and Sons Ltd., London, 1973).

${ }^{4}$ A.Krier, H.Gao, V.Sherstnev, Yu. Yakovlev, Electronics Letters, 351665 (1999)

${ }^{5}$ R. Windisch, B.Dutta, M.Kuijk, A.Knobloch, S.Meinlschmidt, S.Schoberth, P.Kiesel, G.Borghs, G.H.Dohler, and P.Heremans, IEE Transactions on electronic deivces, 471492 (2000)

${ }^{6}$ E.Hadji, J.Bleuse, N.Magnea, and J.L.Pautrat, Appl. Phys. Lett. 672591 (1995)

${ }^{7}$ O.S.Heavens, The optical properties of thin solid films, p.69 (Dover Publications, New York, 1965)

${ }^{8}$ T.Ashley, A.B.Dean, C.T.Elliott, C.F.McConville, G.J.Pryce, and C.R.Whitehouse, Appl. Phys. Lett. 59 1761 (1991)

${ }^{9}$ T.Ashley, C.T.Elliott, N.T.Gordon, R.S.Hall, A.D.Johnson, and G.J.Pryce, Appl. Phys. Lett. 642433 (1994)

${ }^{10}$ J.G.Crowder, C.T.Elliott, and H.R.Hardaway, Electon. Lett. 37116 (2001)

${ }^{11}$ J.G.Crowder - to be published

${ }^{12}$ J.G.Crowder, C.T.Elliott, T.Ashley, A.D.Johnson, and G.J.Pryce, Electron. Lett. 361867 (2000) 